\title{
Tomorrow's world: current developments in the therapeutic use of technology for psychosis ${ }^{\dagger}$
}

\author{
Puffin O'Hanlon, Golnar Aref-Adib, Andres Fonseca, Brynmor Lloyd-Evans, \\ David Osborn \& Sonia Johnson
}

\begin{abstract}
SUMMARY
There is now an established evidence base for the use of information and communication technology (ICT) to support mental healthcare ('e-mental health') for common mental health problems. Recently, there have been significant developments in the therapeutic use of computers, mobile phones, gaming and virtual reality technologies for the assessment and treatment of psychosis. We provide an overview of the therapeutic use of ICT for psychosis, drawing on searches of the scientific literature and the internet and using interviews with experts in the field. We outline interventions that are already relevant to clinical practice, some that may become available in the foreseeable future and emerging challenges for their implementation.
\end{abstract}

\section{LEARNING OBJECTIVES}

- Appreciate the potential uses of technology in the management of psychosis

- Understand the current evidence base for the use of technology in the management of psychosis

- Be aware of challenges involved in the development, evaluation and implementation of technology-based interventions for psychosis

\section{DECLARATION OF INTEREST}

P. O'H. is lead researcher, and S. J. and D. O. are joint chief investigators, on the ARIES study, a development and evaluation study of a selfmanagement smartphone app for early intervention services. A. F. is CEO of an IT company creating digital therapeutics and diagnostics for mental health conditions.

The exponential growth and uptake of information technology (IT) is changing people's everyday lives and how they seek information about their health (OfCom 2015). The opportunity to harness the 'digital revolution' to improve the availability, efficacy, quality and cost-effectiveness of care is emerging as a key focus in policy and practice (Department of Health 2014). Interest and investment in the use of innovative technologies in mental health services is growing. But while web-based (Musiat 2014) and virtual reality (VR) (Opris 2012) interventions have proved effective for common mental disorders, the therapeutic use of information and communication technologies (ICT) for psychosis is less advanced. The underdevelopment of e-mental health for psychosis may reflect uncertainty regarding whether people with psychosis can access or make effective use of technologies. However, recent evidence suggests that people with psychosis are adopting technology in a similar way to the wider population (National Alliance on Mental Illness 2011; Ennis 2012; ArefAdib 2016; Firth 2016) and has promoted interest in the therapeutic use of ICT with this group.

Several ICT-based interventions for psychosis have been developed to date and a number of potential benefits have been proposed (Table 1). Developments include the use of technology as a platform for the delivery of psychosocialintervention content, in a similar way to established e-mental health interventions (e-interventions) for anxiety and depression. However, some applications of technology may be particularly suited to the experience of psychosis and its treatment. For example, creating a VR embodiment of a voice may facilitate learning of more helpful ways of relating to auditory hallucinations, and systems using smartphone-embedded sensors may enable early detection of unique signatures of psychotic relapse.

This overview of the current state of e-interventions for psychosis has been informed by scoping reviews of the published and grey literature and interviews with experts in the field. The interviews, conducted by P. O'H. and G.A.-A., focused on the current state of the field of the therapeutic use of technology for psychosis, its potential benefits and challenges for implementation. We have included only ICTbased interventions designed for the treatment or management of psychosis, and have not considered the myriad other e-mental health resources such
ARTICLE

Puffin O'Hanlon is a researcher at University College London (UCL). She has an MA in Social Anthropology from the University of Cambridge and an MSc in Experimental Psychology from the University of Sussex. Golnar Aref-Adib is a National Institute for Health Research Academic Clinical Fellow at UCL and Camden and Islington NHS Foundation Trust. Andres Fonseca is a consultant psychiatrist with 17 years of clinical experience. $\mathrm{He}$ is on the specialty register for both adult and old age psychiatry. $\mathrm{He}$ is an honorary lecturer at the UCL Division of Psychiatry. Brynmor Lloyd-Evans is a senior lecturer in mental health and social care at UCL. David Osborn is Professor of Psychiatric Epidemiology at UCL and a consultant psychiatrist with the North Camden Crisis

Resolution Team. Sonia Johnson is Professor of Social and Community Psychiatry at UCL and a consultant psychiatrist with the Camden and Islington Early Intervention Service. Correspondence Puffin O'Hanlon, University College London, UCL Division of Psychiatry, 6th Floor, Maple House, 149 Tottenham Court Road, London W1T 7NF, UK. Email: p.hanlon@ucl.ac.uk

Copyright and usage (C) The Royal College of Psychiatrists 2016. This is an open access article distributed under the terms of the Creative Commons Attribution (CC BY) licence.

'See commentary, pp. 311-312, this issue. 


\begin{tabular}{|c|c|c|}
\hline Technology & Potential clinical uses & Potential benefits \\
\hline \multirow[t]{2}{*}{$\begin{array}{l}\text { Clinician-led telephone and } \\
\text { SMS (text message) contact }\end{array}$} & \multirow[t]{2}{*}{ Medication adherence: clinician-led monitoring and remote support } & $\begin{array}{l}\text { Improved access to services for people who are geographically } \\
\text { remote or fear stigma associated with attending services }\end{array}$ \\
\hline & & $\begin{array}{l}\text { Greater flexibility of support and frequent brief contact may } \\
\text { promote engagement for people with low motivation or symptoms } \\
\text { of psychosis or comorbid disorders that interfere with functioning }\end{array}$ \\
\hline \multirow[t]{3}{*}{ Automated SMS systems } & \multirow{3}{*}{$\begin{array}{l}\text { Relapse prevention: monitoring signs of relapse via self-report, } \\
\text { provision of low-level support via automated SMS or triggering a } \\
\text { service-level response }\end{array}$} & Reduced staff time required for intervention delivery \\
\hline & & $\begin{array}{l}\text { Early detection of relapse and more timely provision of support } \\
\text { and intervention }\end{array}$ \\
\hline & & Greater independence in self-care \\
\hline \multirow[t]{3}{*}{$\begin{array}{l}\text { Computer or } \\
\text { web-based programs }\end{array}$} & \multirow{3}{*}{$\begin{array}{l}\text { Delivery of psychosocial intervention content (e.g. cognitive- } \\
\text { behavioural therapy, cognitive remediation therapy and } \\
\text { psychoeducation), with or without staff support }\end{array}$} & $\begin{array}{l}\text { Reduced staff time and level of expertise required for intervention } \\
\text { delivery }\end{array}$ \\
\hline & & Greater standardisation of intervention content \\
\hline & & Greater user choice and control over intervention access \\
\hline \multirow[t]{4}{*}{ Smartphone and tablet apps } & \multirow{4}{*}{$\begin{array}{l}\text { Enhancing routine care: structuring face-to-face clinician-patient } \\
\text { communication } \\
\text { Delivery of self-management interventions, with or without staff } \\
\text { support } \\
\text { Relapse prevention: real-time monitoring of signs of relapse (from } \\
\text { self-report or passively collected data); delivery of self-management } \\
\text { intervention content or triggering a service-level response } \\
\text { Physical health: health and fitness apps }\end{array}$} & $\begin{array}{l}\text { More structured, standardised provision of routine care } \\
\text { Unlimited access to low-level support in situ }\end{array}$ \\
\hline & & $\begin{array}{l}\text { Early detection of relapse and more timely provision of support } \\
\text { and intervention }\end{array}$ \\
\hline & & $\begin{array}{l}\text { Passive collection of data may be more sensitive than self-report, } \\
\text { and more acceptable to some people }\end{array}$ \\
\hline & & \\
\hline Therapeutic serious games & $\begin{array}{l}\text { Enhancing intervention uptake and engagement through } \\
\text { incorporation into technology-based interventions }\end{array}$ & $\begin{array}{l}\text { May promote motivation and engagement with therapy tasks over } \\
\text { the short and long term }\end{array}$ \\
\hline Virtual and augmented reality & $\begin{array}{l}\text { Therapeutic tool in therapist-led or computerised interventions to } \\
\text { provide realistic but controlled learning environments or new ways } \\
\text { of working with hallucinations }\end{array}$ & $\begin{array}{l}\text { May enhance intervention potency, e.g. through promoting the } \\
\text { transfer of therapeutic learning to real-world contexts }\end{array}$ \\
\hline
\end{tabular}

as symptom-assessment methods and patient information websites.

\section{Clinician support via phone calls and SMS}

Interventions consisting of therapeutic contact with a clinician via the telephone or SMS

BOX 1 Telephone and SMS-based (text-based) interventions for psychosis

\section{Clinician-led interventions}

The mobile interventionist (Ben-Zeev 2014a): a mental health professional engages the patient in daily text messages to assess medication adherence and clinical status, provide feedback and support, and suggest coping strategies

Telephone Intervention - Problem Solving (TIPS; Beebe 2004) and telephone medication management (TMM; Salzer 2004): a mental health professional remotely monitors patients during brief weekly phone calls, and provides psychoeducation and support, with problem-solving relating to challenges of community living, including medication adherence, as an adjunct to routine care.

Automated text-messaging systems Information Technology Aided Relapse Prevention Programme in Schizophrenia
(ITAREPS; Španiel 2012): the patient and a family member complete the Early Warning Signs Questionnaire by SMS in response to an automated text message. If the score indicates relapse an automated email alert is sent to the patient's psychiatrist, specifying a protocol for clinician-led response, including telephone contact and a $20 \%$ increase in antipsychotic medication.

Mobile Assessment and Treatment for Schizophrenia (MATS; Granholm 2012): automated text messages are sent daily, using previously gathered information about the individual to personalise questions and responses. Responses incorporate CBT techniques (e.g. thought challenging) and target social interaction, medication adherence and auditory hallucinations. (text messages) may help overcome barriers to accessing support, such as geographical distance or fears of stigmatisation in clinics. Evidencebased interventions showing promise are outlined in Box 1. Telephone or SMS exchanges with a mental health professional - focusing on medication adherence, assistance with problemsolving and coping strategies - appear feasible and may improve outcomes, including medication adherence, patient-clinician rapport and hospital admissions, although more high-quality, wellcontrolled studies are needed (Kasckow 2014). Telephone or SMS support may be more acceptable for some people than face-to-face contact and may have particular utility with high-cost, highrisk groups such as people with co-occurring psychosis and substance misuse. In a small open trial of the 'mobile interventionist' intervention, consisting of daily SMS exchanges with a mental health professional, engagement was high, and participants provided higher therapeutic alliance ratings with the mobile interventionist than with clinicians with whom they had regular face-to-face contact (Ben-Zeev 2014a). Professor Dror Ben-Zeev has advised that these approaches are essentially 'ready to go', given adequate staff training (e.g. in the therapeutic use of text messages) and attention to patient privacy and safety protocols (D. BenZeev, interview with G. A.-A., 2015). 


\section{Automated SMS messaging systems}

Technology may also enable psychosocial intervention independently of costly staff time, potentially allowing services to offer such treatments to a greater number of people. Automated systems may be more resource-efficient, allow realtime assessment and more timely intervention, and promote greater independence in self-care. Automated systems use computer algorithms to encourage users to report their mental state, detect responses that indicate increased risk, and prompt an appropriate self-management or service response. Automated systems have built on the successful use of personal digital assistants (PDAs; offline systems that prompt users to report psychotic experiences at random times throughout the day) to monitor psychotic experiences in real time (Ben-Zeev 2011). Two early automated systems using SMS messages - Mobile Assessment and Treatment for Schizophrenia (MATS), which encourages self-management of psychosis symptoms, and Information Technology Aided Relapse Prevention Programme in Schizophrenia (ITAREPS), which prompts a clinician response to signs of psychosis relapse - are outlined in Box 1.

Preliminary testing suggests that MATS and ITAREPS are feasible and potentially effective, at least for some people with psychosis (van der Krieke 2014). Results from an open trial of MATS $(n=55)$ indicate that automated SMS utilising techniques from cognitive-behavioural therapy (CBT) may be effective in improving social interactions and reducing hallucination severity, but may not be suitable for people with lower functioning and more severe negative symptoms (Granholm 2012). Randomised controlled trial (RCT) results indicate that ITAREPS appeared to reduce hospital admissions, although only when clinicians adhered to the protocol, which they failed to do in $61 \%$ of indicated instances (Španiel 2012).

\section{Enhancing routine care}

The DIALOG+ intervention (Priebe 2015) was designed to structure communication between patients and clinicians in routine meetings in a patient-centred way. Patients use an app on a tablet computer to rate their satisfaction with eight life domains and treatment, and indicate areas in which they need extra support. Current and previous ratings are then displayed on the screen. Clinicians receive brief training in structuring a subsequent discussion using a four-step approach informed by principles of solution-focused therapy. Compared with an active control, a 6-month intervention involving monthly
DIALOG+ sessions led to improved subjective quality of life, fewer unmet needs and better objective social outcomes in a cluster RCT (Priebe 2015). However, implementation was variable, with patients receiving fewer than three of six sessions on average, and 30\% receiving no sessions at all. The DIALOG+ app is freely available for download from the iTunes store, ${ }^{a}$ and a web-based training package for clinicians is available from Priebe et al.

\section{Digitising psychosocial interventions}

As few as 1 in 10 people with psychosis are offered a recommended psychological therapy, and even fewer actually receive an evidence-based psychological intervention (Haddock 2014). A lack of resources, including trained staff and staff time, has been identified as an important barrier to access. Guided computerised CBT self-help interventions for anxiety and depression have been found to be both clinically effective and cost-effective (Musiat 2014), and similar interventions have the potential to facilitate greater access to psychological therapy for people with psychosis (Box 2).

\section{Web-based psychoeducation}

Several web-based psychoeducation interventions have been developed for psychosis. It is an emerging field, with few high-quality studies, but systematic reviews have concluded that web-based psychoeducation about psychosis is feasible, acceptable

\section{BOX 2 Web-based psychological therapies} for psychosis

Coping with Voices (Gottlieb 2013): targets auditory hallucinations, using interactive exercises and games to exemplify CBT principles and behavioural and cognitive coping strategies.

HORYZONS (Alvarez-Jimenez 2013): integrates peer- and clinician-moderated online social networking and tailored interactive web-based psychoeducation modules targeting key recovery domains and risk factors for psychotic relapse for patients with first-episode psychosis.

MieliNet (Anttila 2012): information about schizophrenia, its symptoms and patients' rights, a chat room for peer support, and a $0 \&$ A column. Designed to structure a psychoeducation intervention delivered by trained nursing staff over six sessions.

SOAR (Schizophrenia Online Access to Resources) (Rotundi 2010): psychoeducation for people with schizophrenia and their supporters through self-guided psychoeducational materials and therapist-moderated online forums. a. We are referring to DIALOG+, which is included in the DIALOG app developed by Kleomenis Katevas. Confusingly, there are several apps named 'dialog' (in various combinations of upper and lower case letters). 
and potentially effective for people of various ages and stages of illness (Välimäki 2012; AlvarezJimenez 2014; van der Krieke 2014). Challenges include engagement with self-guided interventions, which is also a problem in evaluations of unsupported web-based interventions for common mental health problems (Kaltenthaler 2008).

In in-patient settings, web-based psychoeducation packages could support patients' selfmanagement skills and nurses' psychoeducation skills, and facilitate greater consistency of treatment across settings. A feasibility study in which participants on an acute in-patient ward used the website MieliNet (Box 2) with nurse support found that $75 \%$ of the sessions were completed (Anttila 2012). However, patients' mental state and level of insight, and patients' and nurses' IT skills affected completion rates, indicating that although the majority of acutely unwell patients were able to use the intervention, computerised interventions may not be acceptable for all.

In community settings, computerised interventions could facilitate increased access to recommended psychosocial interventions and greater user choice and control over when and where they receive it. A small pilot RCT of a family intervention using the SOAR website (Box 2) delivered in patients' homes with minimal therapist input reported high engagement with the intervention, and significant improvements in positive symptoms and knowledge about schizophrenia compared with usual care (Rotundi 2010). HORYZONS, a webbased relapse prevention intervention for patients with first-episode psychosis (Box 2), was found to be acceptable, feasible and safe when delivered to young people with little clinician support in an open trial, although only $20 \%$ of participants completed at least five of seven psychoeducation modules (Alvarez-Jimenez 2013).

\section{Computerised CBT}

Cognitive-behavioural therapy for psychosis (CBT-P) targets negative appraisals of psychotic experiences in order to reduce associated distress and improve functioning. The National Institute for Health and Care Excellence (NICE) recommends access to CBT-P for everyone with a schizophrenia spectrum diagnosis, but current provision is poor (Haddock 2014). Low-intensity CBT interventions may help to close this gap, although we found just one evaluation of a web-based CBT-P intervention. In a small open study, Coping with Voices (Box 2) was found to be acceptable and feasible for adults when delivered without support in a mental health setting, and significant pre-post reduction in voice severity was observed for those who completed the intervention (Gottlieb 2013). However, only 25\% of participants reported following through with homework assignments. A larger trial is underway (see http://cpr.bu.edu/research/current-research/ coping-with-voices).

\section{Computer-assisted cognitive remediation therapy}

Cognitive remediation therapy (CRT) is a behavioural-training-based intervention targeting cognitive deficit. It aims to enhance cognitive processes (attention, memory, executive function, social cognition and metacognition), with the ultimate goal of functional rehabilitation, and has been found to be modestly effective (Wykes 2011). CRT traditionally involved manual tasks, but computer-based training has also been used since the 1990s. The use of computer software allows unlimited task repetitions, a variety of forms of reinforcement, accurate and instantaneous feedback, automatic adjustment of difficulty level based on performance (Grynszpan 2011), and personalised yet standardised treatment. Computer-assisted cognitive remediation therapy (CACRT) has the most established evidence base of any intervention for psychosis using IT: metaanalyses have found similar magnitudes of effect to manualised CRT (Grynszpan 2011).

Traditionally, CRT involves multiple repetitions of abstract cognitive tasks, which can be tedious and require adjunctive rehabilitative work to generalise the benefits of training to improvements in daily functioning (Reeder 2016). A new computerised CRT package, CIRCuiTS, is designed to enable this skills transfer to take place within the training program itself, through the use of both abstract tasks and simulated activities of daily living (e.g. food shopping) in a virtual village environment. Results of early feasibility testing suggest that this format is highly acceptable to users (Reeder 2016).

Although the majority of CRTs (whether computer-assisted or not) involve considerable face-to-face contact, the possibilities afforded by computerised training packages may enable more cost-effective interventions, through reducing the therapist time and expertise required to deliver them. Professor Til Wykes and colleagues are currently exploring the web-based CIRCuiTS package in a clinical setting with health worker support; exact requirements for therapist time and skill are yet to be determined (T. Wykes, interview with P. O'H, 2015).

\section{Future directions}

\section{Apps}

Evidence suggests that people with psychosis increasingly own smartphones (Firth 2016). As in the general population, ownership seems to 
be higher among younger people, with reports of around $69 \%$ of patients with first-episode psychosis owning an internet-enabled mobile device (Lal 2015). More akin to a computer than their mobile phone predecessors, but usually carried on the person, smartphones provide a promising way of assessing need for support in real time and delivering timely, interactive interventions making use of web-based resources, audio/visual content and other functionality - as people go about their everyday lives (Danaher 2015). Although there is currently little empirical evidence to support their implementation, several apps for psychosis have been developed (Box 3), and results from five studies of four smartphone apps suggest that they are acceptable and feasible (Firth 2015).

Smartphone intervention delivery may be particularly helpful for people with psychosis, since cognitive impairment and motivational difficulties may limit the ability to remember, initiate and maintain strategies learned in therapy sessions. To the best of our knowledge, the FOCUS app (Box 3) is the only psychosocial app intervention to have been evaluated to date. In an open trial, patients reported feeling confident, comfortable and satisfied with the FOCUS app, and usage was high - 5 times per day on average. Significant reductions were found on scores for depression and positive symptoms of schizophrenia after using the app for 1 month (Ben-Zeev 2014b). Ben-Zeev and colleagues are now conducting a large RCT (trial identifier: NCT02421965) comparing FOCUS with clinic-based self-management for adults with serious mental illness. A CBT-informed app for patients with first-episode psychosis, Actissist (Box 3), is also currently being evaluated in an RCT (Bucci 2015).

Smartphone apps might also be used to enhance automated intervention models. Self-reporting of psychotic symptoms via ClinTouch (Box 3) not only produces clinically meaningful data, but also seems to increase adherence compared with SMS methods (Ainsworth 2013). A trial is currently underway (trial identifier: ISRCTN88145142; chief investigator: Dr Eve Applegate) of an automated system using ClinTouch that alerts both patients and their clinical team in real time to indications of relapse in order to prompt early intervention.

Qualitative data from the ClinTouch studies suggest that some patients find the regular reporting of symptoms to be repetitive, which may lead to disengagement in the longer term (Palmier-Claus 2013). Researchers are currently investigating whether passive monitoring of indicators of psychotic relapse using the sensor technologies increasingly embedded in smartphones (e.g. accelerometers, the Global Positioning System

(GPS), microphones) may be more acceptable to users and more sensitive to change than selfreport. The Crosscheck system is currently being developed and tested to detect patterns in paralinguistic voice properties, physical activity, location, sleep patterns, mood and psychotic symptoms, and to generate personalised early warning models (trial identifier: NCT01952041; princial investigator: Dr Dror Ben-Zeev). An early prototype of the system seems to be acceptable to participants with psychosis, although the research team note that self-selection is likely, with those who are concerned about this type of monitoring choosing not to take part in testing (D. BenZeev, interview with G.A.-A., 2015). If successful, Crosscheck could be used to flag up potential relapse and prompt an early intervention response in a similar way to ClinTouch and ITAREPS.

Smartphone apps might also tacklethe prevalence of obesity and cardiometabolic disorders among people with psychosis (which is twice that in the general population (Osborn 2008)). In the general population, fitness apps are growing in popularity more than any other app category (Khalaf 2014), and results from three small feasibility studies of fitness apps for people with serious mental illness are promising (Naslund 2015).

B0X 3 Smartphone apps for psychosis

Actissist (not yet available to the public) (Bucci 2015; http://sites.psych-sci. manchester.ac.uk/actissist): prompts users three times a day to answer self-assessment questions, followed by messages containing normalisation and CBT strategies for managing distress in five domains: perceived criticism, socialisation, cannabis use, paranoia and distressing voices. Additional multimedia psychoeducational content.

ClinTouch (Palmier-Claus 2013; www. clintouch.com): prompts users to answer questions tailored to their symptoms of psychosis and depression using computer algorithms and feeds information to a clinical database accessible to clinicians, prompting discussion during routine appointments or an alert for additional service response.

FOCUS (not yet available to the public) (Ben-Zeev 2014b): prompts users to answer daily self-assessment questions (related to medication, voices, mood, social interaction and sleep), and encourages users to engage in illness self-management strategies structured as $0 \& A$ exchanges, advice and visual aids, drawing on CBT principles, social skills training and illness management.

My Journey (Surrey and Borders NHS Foundation Trust; available free from Google Play): psychotic experiences, mood, sleep, stress, alcohol and cannabis use, medication trackers and advice for each area, and information about psychosis. Reminder function for activities and coping strategies. Under development to incorporate recovery and relapse-prevention planning tools.

Silver Linings (Birmingham and Solihul NHS Foundation Trust; free from Google Play): mood, stress, sleep, activity, psychotic experiences and medication trackers and advice for each area. Visual displays of ratings over time can be shared with clinicians. Medication and appointment reminder function, 'gamification' rewards system.

WellWave (Macias 2015; www.wellframe. com): delivers daily menu of suggested activities, including exercise prompts, regular self-assessments of physical health quality, health control and stage of exercise, and passive recording of physical activity. 


\section{Therapeutic serious games}

Serious games are video games with a primary purpose other than pure entertainment. Their potential lies in their ability to increase engagement with treatment that is painful (e.g. chemotherapy) or boring (e.g. repetitive rehabilitation tasks) (Kato 2010). Techniques that have been successful in engaging people in commercial games - including daily 'quests' and reward systems such as 'boosters' or extra lives - could be used to increase adherence to e-interventions for psychosis. Serious games designed for physical health complaints have shown promise (Kato 2010).

In psychosis, gaming techniques might enhance motivation to complete repetitive tasks targeting cognition (Chan 2010). A study of cognitive training for adults with schizophrenia or schizoaffective disorder found that creating a personalised fantasy gaming context for training tasks led to greater intrinsic motivation to complete the tasks, improved attention and enhanced task-related learning (Chan 2010). Bias Buster, a recently developed serious game for patients with first-episode psychosis, is currently under investigation in an RCT (L. van der Krieke, interview with G. A.-A., 2015). In this version of a popular commercial game, the user earns bonuses by completing repetitive cognitive bias modification (CBM) computer tasks. CBM has shown promise in targeting cognitive biases implicated in the development of psychosis (Steel 2010), but engagement has been identified as a potential challenge. Bias Buster was found to be acceptable to a small sample of patients with firstepisode psychosis, although it fell short of their expectations of a video game (Wartena 2013). More investigation is needed into how to present serious games to people with psychosis, as well as which psychosocial approaches they might add value to and for whom.

\section{Virtual and augmented reality \\ Virtual reality}

Virtual reality gives a sense of being immersed and present in, and interacting with, a threedimensional world. This experience can elicit similar psychological and physiological responses to the real world. VR technology seems to be acceptable, safe and engaging for people with psychosis (Macedo 2014), and potential applications in assessment and treatment are beginning to be explored (Freeman 2008).

The immersive nature of VR experiences offers opportunities for therapeutic learning, skills practice and confidence-building in a low-threat environment for people with psychosis for whom perceived threat or hospital admission make realworld encounters difficult. Social skills training using VR role-play may enhance motivation and lead to greater improvements in conversational and assertiveness skills than traditional roleplay (Park 2011). The participant wears a headmounted display and a position tracker, and has the experience of being immersed in a virtual environment (e.g. a coffee shop) through their own avatar, and interacting with other avatars, who give corrective feedback according to the person's interaction during a conversation role-play.

VR technology might also enhance cognitive behavioural interventions for psychosis (Freeman 2008). Behavioural experiments that challenge threat beliefs are considered an essential component of CBT for psychosis, but can be unacceptably daunting for patients in real-world contexts. Professor Daniel Freeman is currently undertaking a small exploratory RCT of a VR intervention for paranoia, and reports indications of positive outcomes (D. Freeman, interview with P. O'H, 2015).

VR has recently been used in the development of AVATAR therapy. People who are able to have dialogues with their voices feel more in control and less helpless than those who are not, but establishing a dialogue with an entity you cannot see presents particular challenges (Leff 2014). In AVATAR therapy, participants are able to create a virtual embodiment of their voice (Fig. 1). A therapist both speaks through the avatar, and provides encouragement for the individual to engage in a dialogue with it. AVATAR therapy aims to enable the individual to learn to gain control over the avatar, with the intention that they will generalise this experience to their persecutory voice.

Results from a recent proof-of-concept study of AVATAR therapy are promising (Leff 2013) and a larger RCT of AVATAR therapy is currently underway (Craig 2015). Professor Tom Craig, who

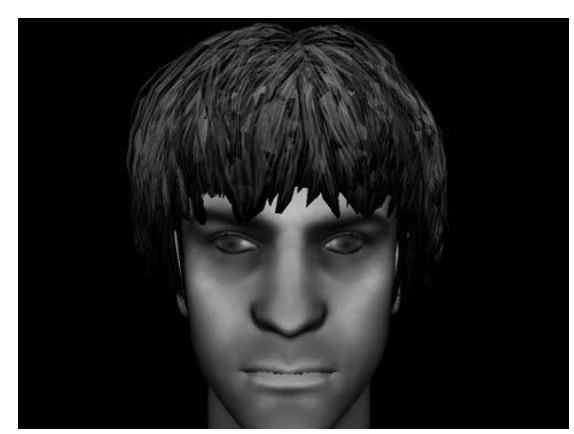

An example avatar of a participant's persecutory voice, from AVATAR therapy. 
leads the AVATAR trial, has commented that VR gives 'one sort of handle on psychotic experience that we've never had before' and may enhance intervention potency (T. Craig, interview with P.O'H., 2015). Since AVATAR therapy requires significant therapist expertise and time, and uses expensive equipment, future research will need to demonstrate positive effects on realworld outcomes in definitive trials, and weigh up effectiveness against the cost of delivery.

\section{Augmented reality}

Augmented reality is a perception of the real world that is augmented or supplemented by computergenerated stimuli. Computer-generated graphics and sounds are merged with objects and/or locations in the real world, and/or information in the form of text or speech is overlayed onto the user's visual field. This enhances the real-world environment, rather than providing an immersive experience in a virtual one. For example, Google Glass overlays a virtual map onto the user's realworld view, with directions, street names, shop locations and other information displayed as floating text.

Augmented reality has received some attention in graded exposure interventions for specific phobias (Baus 2014) and could be harnessed in interventions targeting hallucinations or cognitive and social functioning. For example, augmented reality could be used to deliver components of social rehabilitation or CRT in real-world contexts by providing reminders, instructions and support in situ.

\section{Challenges}

A number of practical and ethical issues have been described in relation to e-interventions in psychiatry, including equipment and software set-up and maintenance costs, the ability of IT infrastructures in healthcare to accommodate novel technologies, data protection, and patient privacy, safety and security (e.g. Hollis 2015). A few challenges are especially relevant to the future development, evaluation and use of e-interventions for psychosis.

\section{Building an evidence base}

First, clinical effectiveness and cost-effectiveness have hardly been addressed by existing studies. Since we know so little about the digital lives of people with psychosis, and in light of evidence that characteristics such as cognitive deficit and disorganised thinking can make navigating mainstream user interfaces difficult (Rotundi 2010), research to date has focused on establishing feasibility and usability of interventions. There is now substantial evidence from small-scale feasibility studies that people with psychosis are interested in and able to use a diverse array of digital technology interventions designed to accommodate their particular needs (Naslund 2015). However, with technology advancing at a rate that far outpaces the time lines of RCTs, there is a risk that the technology underpinning these interventions will become outdated before their efficacy has been investigated. For example, with smartphone manufacturers releasing upwards of two new models a year, smartphone functionality will have advanced six times during the lifespan of a definitive RCT. People's expectations are likely to be shaped by their experience of rapidly advancing technology in other parts of their lives - as evidenced by participants' feedback for Bias Buster - and this is likely to affect the acceptability of digital interventions.

Developers and researchers should seek to incorporate emerging technologies into novel interventions, to progress as quickly as possible to trialling them in adequately powered RCTs, and to rapidly disseminate findings from development and evaluation stages in order to keep up with technological developments.

\section{Engaging the patient}

Second, early indicators of poor engagement are emerging from studies of low-intensity e-interventions for psychosis (e.g. Gottlieb 2013). There is some evidence to suggest that interactive technology, including gaming techniques (Chan 2010) and VR (Park 2011), may enhance participants' engagement and motivation. However, substantial evidence suggests that supported selfhelp interventions - including e-interventions - for common mental health problems are more effective (Musiat 2014) and associated with higher rates of completion (Richards 2012) than unsupported interventions, and this is likely to be the case for emerging e-interventions for psychosis. It is less clear how the type, amount or mode of delivery (e.g. face-to-face, telephone or online) of support or who provides it (e.g. mental health professional or support worker) affect outcomes (Musiat 2014), and this will need attention in future research into e-interventions for psychosis. The existing and emerging technologies discussed here including sensor technologies, gaming and VR - could be blended in future interventions with technology-enabled human support (e.g. via SMS, email or moderated forums) to develop novel ICT interventions with tailored, timely and engaging content and low-intensity therapeutic contact. 
MCO answers

$1 \mathrm{~b} \quad 2 \mathrm{a} \quad 3$ e 4 e $\quad 5 \mathrm{~b}$

\section{Engaging the clinician}

Third, digitised methods must gain acceptance among clinicians if they are to be implemented successfully. Findings from the ITAREPS trial suggested that many treating psychiatrists failed to adhere to the treatment protocol because they lacked confidence in a system of assessment based on rating scales and IT. Qualitative data from the DIALOG+ trial are not yet available, but findings of poor clinician adherence to an intervention that requires minimal training and no service reorganisation (Priebe 2015) highlight the importance of understanding and addressing likely implementation challenges for more complex technology-based treatments.

Clinician involvement in the development and evaluation of digital tools, and adequate support to promote the uptake of novel interventions, will be essential for the successful incorporation of new technologies in clinical practice.

\section{Barriers to access}

Finally, e-interventions could exacerbate inequalities in service provision. Technology can only extend the reach of services if patients can access and engage with it. Access to technology does appear to be reduced in people with psychosis compared with the general population, and especially among people who are older, of a Black or ethnic minority background, have lower income and experience greater functional impairment (Ennis 2012; Firth 2015). Wide roll-out of e-interventions without additional support for these groups would risk further marginalisation. Furthermore, neither access nor interest is the same as engagement, and we need a better understanding of how income, educational level, ethnicity and age affect the digital lives of people with psychosis and their ability to use e-interventions effectively.

\section{Conclusions}

Information and communication technologies enable the delivery of interactive, evidence-based interventions for the prevention and management of psychosis in real-world contexts. The therapeutic use of technology for psychosis has the potential to increase access to standardised evidence-based treatments (such as psychological therapies), enable greater patient choice and control, and foster greater independence through self-care. E-mental health may not be acceptable or helpful for everyone, but interventions making use of the internet, serious gaming, augmented reality or total immersion in a virtual world could dramatically change mental healthcare for psychosis. There is substantial evidence that such interventions are acceptable to and usable by people with psychosis from a range of backgrounds and age groups. We now need to determine whether, and for whom, they are clinically effective and cost-effective and how best to integrate them into existing healthcare systems.

\section{Funding}

The research informing this article was supported by the National Institute for Health Research (NIHR) Collaboration for Leadership in Applied Health Research and Care North Thames at Barts Health NHS Trust. The views expressed are those of the authors and not necessarily those of the NHS, the NIHR or the Department of Health.

\section{Acknowledgements}

The authors would like to thank Professor Dror Ben-Zeev, Professor Tom Craig, Professor Daniel Freeman, Professor Stefan Priebe, Dr Lian van der Krieke and Professor Til Wykes for taking part in interviews that informed the content of this article.

\section{References}

Ainsworth J, Palmier-Claus JE, Mach M, et al (2013) A comparison of two delivery modalities of a mobile phone-based assessment for serious mental illness: native smartphone application vs text-messaging only implementations. Journal of Medical Internet Research, 15(4): e60.

Alvarez-Jimenez M, Bendall S, Lederman R, et al (2013) On the HORYZON: moderated online social therapy for long-term recovery in first episode psychosis. Schizophrenia Research, 143: 143-9.

Alvarez-Jimenez M, Alcazar-Corcoles MA, Gonzalez-Blanch C, et al (2014) Online, social media and mobile technologies for psychosis treatment: a systematic review on novel user-led interventions. Schizophrenia Research, 156: 96-106.

Anttila M, Välimäki M, Hätönen $H$, et al (2012) Use of web-based patient education sessions on psychiatric wards. International Journal of Medical Informatics, 81: 424-33.

Aref-Adib G, O'Hanlon P, Fullarton K, et al (2016) A qualitative study of online mental health information seeking behaviour by those with psychosis. BMC Psychiatry, 16: 232.

Baus 0, Bouchard S (2014) Moving from virtual reality exposure-based therapy to augmented reality exposure-based therapy: a review. Frontiers in Human Neuroscience, 8: 112

Beebe LH, Tian L (2004) TIPS: Telephone Intervention - Problem Solving for persons with schizophrenia. Mental Health Nursing, 25: 317-29.

Ben-Zeev D, Morris S, Swendsen J, et al (2011) Predicting the occurrence, conviction, distress, and disruption of different delusional experiences in the daily life of people with schizophrenia. Schizophrenia Bulletin, 37: 1248-56.

Ben-Zeev D, Kaiser SM, Krzos I (2014a) Remote "hovering" with individuals with psychotic disorders and substance use: feasibility, engagement, and therapeutic alliance with a text-messaging mobile interventionist. Journal of Dual Diagnosis, 10: 197-203.

Ben-Zeev D, Brenner CJ, Begale M, et al (2014b) Feasibility, acceptability, and preliminary efficacy of a smartphone intervention for schizophrenia. Schizophrenia Bulletin, 40: 1244-53.

Bucci S, Barrowclough C, Ainsworth J, et al (2015) Using mobile technology to deliver a cognitive behaviour therapy-informed intervention in early psychosis (Actissist): study protocol for a randomised controlled trial. Trials, 16: 404. 
Chan CLF, Ngai EKY, Leung PKH, et al (2010) Effect of the adapted virtual reality cognitive training program among Chinese older adults with chronic schizophrenia: a pilot study. International Journal of Geriatric Psychiatry, 25: 643-9.

Craig TKJ, Rus-Calafell M, Ward T, et al (2015) The effects of an Audio Visual Assisted Therapy Aid for Refractory auditory hallucinations (AVATAR therapy): study protocol for a randomised controlled trial. Trials, 16: 349 .

Danaher BG, Brendryen H, Seeley JR, et al (2015) From black box to toolbox: outlining device functionality, engagement activities, and the pervasive information architecture of mHealth interventions. Internet Interventions, 2: 91-101

Department of Health (2014) Closing the Gap: Priorities for Essential Change in Mental Health. Department of Health.

Ennis L, Wykes T (2012) Can't surf, won't surf: the digital divide in mental health. Journal of Mental Health, 21: 395-403.

Firth J, Torous J (2015) Smartphone apps for schizophrenia: a systematic review. JMIR mHealth and uHealth, 2(4): e102.

Firth J, Cotter J, Torous J, et al (2016) Mobile phone ownership and endorsement of "mHealth" among people with psychosis: a metaanalysis of cross-sectional studies. Schizophrenia Bulletin, 42: 448-55.

Freeman D (2008) Studying and treating schizophrenia using virtual reality: a new paradigm. Schizophrenia Bulletin, 34: 605-10.

Gottlieb JD, Harper Romeo K, Penn D, et al (2013) Web-based cognitive behavioural therapy for auditory hallucinations in persons with psychosis: a pilot study. Schizophrenia Research 145: 82-7.

Granholm E, Ben-Zeev D, Link PC, et al (2012) Mobile assessessment and treatment for schizophrenia (MATS): a pilot trial of an interactive text-messaging intervention for medication adherence, socialisation and auditory hallucinations. Schizophrenia Bulletin, 38: 414-25.

Grynszpan 0, Perbal S, Pelissolo A, et al (2011) Efficacy and specificity of computer-assisted cognitive remediation in schizophrenia: a metaanalytical study. Psychological Medicine, 41: 163-73.

Haddock G, Eisner E, Boone C, et al (2014) An investigation of the implementation of NICE- recommended CBT interventions for people with schizophrenia. Journal of Mental Health, 23: 162-5.

Hollis C, Morriss R, Martin J, et al (2015) Technological innovations in mental healthcare: harnessing the digital revolution. British Journal of Psychiatry, 206: 263-5.

Kaltenthaler E, Parry G, Beverley C, et al (2008) Computerised cognitivebehavioural therapy for depression: systematic review. British Journal of Psychiatry, 193: 181-4.

Kasckow J, Felmet K, Appelt C, et al (2014) Telepsychiatry in the assessment and treatment of schizophrenia. Clinical Schizophrenia \& Related Psychoses, 8: 21-7.

Kato PM (2010) Video games in health care: closing the gap. Review of General Psychology, 14: 113-21.

Khalaf $S$ (2014) Health and fitness apps finally take off, fueled by fitness fanatics. Flurry Insights [blogl, 19 June. Flurry Analytics (http://mobile. tumblr.com/post/115192181465/health-and-fitness-apps-finally-take-offfueled). Accessed 17 July 2016

Lal S, Dell'Elce J, Tucci N, et al (2015) Preferences of young adults with first-episode psychosis for receiving specialised mental health services using technology: a survey study. Journal of Mental Health, 2(2): e18.

Leff J, Williams G, Huckvale MA, et al (2013) Computer-assisted therapy for medication-resistant auditory hallucinations: proof-of-concept study. British Journal of Psychiatry, 202: 428-33.

Leff J, Williams G, Huckvale M (2014) Avatar therapy for persecutory auditory hallucinations: what is it and how does it work? Psychosis, 6 : $166-76$

Macedo M, Marques A, Quieros C (2014) Virtual reality in the assessment and treatment of schizophrenia: a systematic review. Jornal Brasileiro de Psiquiatria, 64: 70-81.

Macias C, Panch T, Hicks YM, et al (2015) Using smartphone apps to promote psychiatric and physical well-being. Psychiatric Quarterly, 86 : $505-19$.
Musiat P, Tarrier N (2014) Collateral outcomes in e-mental health: a systematic review of the evidence for added benefits of computerized cognitive behaviour therapy interventions for mental health. Psychological Medicine, 44: 3137-50.

Naslund JA, Marsch LA, McHugo GJ, et al (2015) Emerging mHealth and eHEalth interventions for serious mental illness: a review of the literature. Journal of Mental Health, 24: 320-31.

Naslund JA, Aschbrenner KA, Bartels SJ (2016) Wearable devices and smartphones for activity tracking among people with serious mental illness. Mental Health and Physical Activity, 10: e17.

National Alliance on Mental Illness (2011) First Episode: Psychosis Results from a 2011 NAMI Survey. NAMI.

Ofcom (2015) Adults' Media Use and Attitudes: Report 2015. Ofcom

Opris D, Pintea S, Garcia-Palacios A, et al (2012) Virtual reality exposure therapy in anxiety disorders: a quantitative meta-analysis. Depression and Anxiety, 29: 85-93.

Osborn DPJ, Wright CA, Levy G, et al (2008) Relative risk of diabetes, dyslipidaemia, hypertension and the metabolic syndrome in people with severe mental illnesses: systematic review and metaanalysis. BMC Psychiatry, 8: 84.

Palmier-Claus JE, Rogers A, Ainsworth J (2013) Integrating mobile-phone based assessment for psychosis into people's everyday lives and clinical care: a qualitative study. BMC Psychiatry, 13: 34

Park K, Ku J, Choi S, et al (2011) A virtual reality application in role-plays of social skills training for schizophrenia: a randomised, controlled trial. Psychiatry Research, 189: 166-72.

Priebe S, Kelley L, Omer S (2015) The effectiveness of a patient-centred assessment with a solution- focused approach (DIALOG+) for patients with psychosis: a pragmatic cluster-randomised controlled trial in community care. Psychotherapy and Psychosomatics, 84: 304-13.

Reeder C, Pile V, Crawford P, et al (2016) The feasibility and acceptability to service users of CIRCuiTS, a computerized cognitive remediation therapy programme for schizophrenia. Behavioural and Cognitive Psychotherapy, 44: 288-305.

Richards D, Richardson T (2012) Computer-based psychological treatments for depression: a systematic review and meta-analysis. Clinical Psychology Review, 32: 329-42.

Rotundi AJ, Anderson CM, Haas GL (2010) Web-based psychoeducational intervention for persons with schizophrenia and their supporters: oneyear outcomes. Psychiatric Services, 61: 1099-105.

Salzer MS, Tunner T, Charney NJ (2004) A low-cost, telephone intervention to enhance schizophrenia treatment: a demonstration study. Schizophrenia Research, 66: 75-6.

Španiel F, Hrdlička J, Novák T (2012) Effectiveness of the information technology-aided program of relapse prevention in schizophrenia (ITAREPS): a randomized, controlled, double-blind study. Journal of Psychiatric Practice, 18: 269-80

Steel C, Wykes T, Ruddle A, et al (2010) Can we harness computerised cognitive bias modification to treat anxiety in schizophrenia? A first step highlighting the role of mental imagery. Psychiatry Research, 178 : 451-5.

Välimäki M, Hätönen H, Lahti M (2012) Information and communication technology in patient education and support for people with schizophrenia. Cochrane Database of Systematic Reviews, 10: CD007198.

van der Krieke L, Wunderink L, Emerencia AC (2014) E-mental health self-management for psychotic disorders: state of the art and future perspectives. Psychiatric Services, 65: 33-49.

Wartena B, van Dijk H (2013) Bias Blaster - aiding cognitive bias modification-interpretation through a bubble shooter induced gameflow. In Games for Health: Proceedings of the 3rd European Conference on Gaming and Playful Interaction in Health Care (eds B Schouten, S Fedtke, T Bekker, et al): 47-60. Springer Verlag.

Wykes T, Huddy V, Cellard C, et al (2011) A meta-analysis of cognitive remediation for schizophrenia: methodology and effect sizes. American Journal of Psychiatry, 168: 472-85. 


\section{MCOs}

Select the single best option for each question stem

1 Evidence suggests that staff support in e-interventions for psychosis:

a should always be delivered by a qualified therapist

b is likely to increase patients' engagement

c is not necessary

$\mathrm{d}$ is only likely to be helpful if delivered face-toface

e is easily implemented in clinical practice.

2 The use of computer-assisted cognitive remediation therapy (CACRT) in psychosis:

a has magnitudes of effect similar to those of manualised CRT b allows only limited task repetitions

$\mathrm{c}$ is a poorly standardised treatment

$d$ is less effective than manualised CRT

e has a poorly established evidence base.

3 Smartphone apps for psychosis have been developed to assess:

a psychotic symptoms and experiences

b patterns in paralinguistic voice properties

c levels of physical activity

d patterns of sleep

e all of the above.

\section{Avatar therapy:}

a aims to teach people distraction techniques to cope with voice hearing

b targets paranoid delusions c is a low-cost alternative to CBT for psychosis

$\mathrm{d}$ can be delivered by unqualified mental health staff with minimal training

e aims to help people to establish a dialogue with their persecutory voice.

5 Internet and mobile-based interventions for psychosis:

a have been shown to be unacceptable to patients

b have the potential to improve clinical and social outcomes

c have been shown to be unfeasible

$d$ have been effective in therapeutic serious gaming only

e have the potential to improve social outcomes only. 